\title{
Magnetite-based magnetoreception in birds: the effect of a biasing field and a pulse on migratory behavior
}

\author{
Wolfgang Wiltschko ${ }^{1, *}$, Ursula Munro ${ }^{2}$, Roswitha Wiltschko ${ }^{1}$ and Joseph L. Kirschvink ${ }^{3,4}$ \\ ${ }^{1}$ Fachbereich Biologie und Informatik, Zoologie, J. W. Goethe-Universität Frankfurt am Main, Siesmayerstrasse 70, \\ D 60054 Frankfurt am Main, Germany, ${ }^{2}$ Department of Environmental Sciences, University of Technology, Sydney, \\ PO Box 123, Broadway, NSW 2007, Australia, ${ }^{3}$ Division of Geological and Planetary Science, The California \\ Institute of Technology, Pasadena, CA 91125, USA and ${ }^{4}$ Department of Earth and Planetary Sciences, University of \\ Tokyo, Hongo Campus, Tokyo, Japan \\ *Author for correspondence (e-mail: wiltschko@zoology.uni-frankfurt.de)
}

Accepted 9 July 2002

\section{Summary}

To test the hypothesis that single domain magnetite is involved in magnetoreception, we treated Australian silvereyes Zosterops $l$. lateralis with a strong, brief pulse designed to alter the magnetization of single domain particles. This pulse was administered in the presence of a $1 \mathrm{mT}$ biasing field, either parallel to the direction of the biasing field (PAR group) or antiparallel (ANTI group). In the case of magnetoreceptors based on freely moving single domain particles, the PAR treatment should have little effect, whereas the ANTI treatment should cause remagnetization of the magnetite particles involved in a receptor and could produce a maximum change in that receptor's output for some receptor configurations. Migratory orientation was used as a criterion to assess the effect on the receptor. Before treatment, both groups preferred their normal northerly migratory direction.
Exposure to the biasing field alone did not affect their behavior. Treatment with the pulse in the presence of the biasing field caused both the PAR and the ANTI birds to show an axial preference for the east-west axis, with no difference between the two groups. Although these results are in accordance with magnetite-based magnetoreceptors playing a role in migratory orientation, they do not support the hypothesis that single domains in polaritysensitive receptors are free to move through all solid angles. Possible interpretations, including other arrangements of single domains and superparamagnetic crystals, are discussed.

Key words: magnetoreception, single domain magnetite, superparamagnetic particles, pulse, biasing field, migratory orientation, Zosterops l. lateralis, Australian silvereye.

\section{Introduction}

The discovery of biogenic magnetite in the radula teeth of chitons (Lowenstam, 1962) and in bacteria (Blakemore, 1975) led to speculation about magnetite $\left(\mathrm{Fe}_{3} \mathrm{O}_{4}\right)$ crystals being involved in magnetoreception. Involvement of these crystals became more likely when magnetite was also found in animal species that were known to orient with the help of the magnetic field (e.g. Gould et al., 1978; Walcott et al., 1979) (for a review, see Kirschvink et al., 1985). Various types of possible receptor using magnetite have been described on the basis of different functional principles, some of which involve single domains and others, superparamagnetic particles (e.g. Yorke, 1979; Kirschvink and Gould, 1981; Kirschvink, 1989; Shcherbakov and Winklhofer, 1999). Several authors tried to verify the role of magnetite-based receptors in orientation processes by behavioral experiments. The first attempts produced inconclusive results (e.g. Gould et al., 1980; Walcott et al., 1988); later ones were more promising (Kirschvink and Kobayashi-Kirschvink, 1991; Kirschvink et al., 1997).

Birds have always been of particular interest for the study of magnetoreception because they rely strongly on the Earth's magnetic field for orientation and navigation. They appear to use information from the geomagnetic field in two ways, namely (1) as a compass for direction finding and (2) as part of their navigational 'map' for determining positions (for a review, see R. Wiltschko and Wiltschko, 1995). These probably involve separate receptor systems with different types of receptor cells, as the biophysical constraints on them differ markedly (e.g. Kirschvink and Walker, 1985). In birds, magnetite was found in the head, particularly in the ethmoid region above the beak (Walcott et al., 1979; Beason and Nichols, 1984; Beason and Brennan, 1986; Kirschvink and Walker, 1986; Edwards et al., 1992) and in the cutis of the upper mandible (Hanzlik et al., 2000; Winklhofer et al., 2001). These parts of the head are innervated by the ophthalmic nerve, a branch of the nervus trigeminus; electrophysiological recordings from this nerve and from the trigeminal ganglion of Bobolinks Dolichonyx oryzivorus (Icteridae) revealed units that responded to changes in the intensity of the magnetic field 
(e.g. Beason, 1989; Semm and Beason, 1990). Similar work in fish also confirmed the role of the ophthalmic nerve and led to the ultrastructural identification of a cell containing singledomain magnetite, which could serve as the much sought-after magnetoreceptor (Walker et al., 1997; Diebel et al., 2000). In view of these findings, it seemed promising to look for a possible involvement of magnetite-based receptors in the orientation processes of birds by behavioral studies.

Since the magnetite particles found in birds appeared to be single domains (Walcott et al., 1979; Kirschvink and Walker, 1986; Beason and Brennan, 1986; Beason, 1989; Edwards et al., 1992), the easiest approach seemed to be to alter the magnetization of these particles with a brief but strong magnetic pulse. If they were involved in receptive processes, this could lead to a dramatic change in the information they mediated, which, in turn, should cause a marked change in the birds' orientation behavior. In studies performed by some of the authors (U.M., R.W., W.W.), treatment with a strong pulse had a considerable effect on the migratory orientation of Australian silvereyes, Zosterops lateralis, deflecting the birds' headings from their natural migratory direction by approximately $90^{\circ}$ towards the east for about 2 days (W. Wiltschko et al., 1994, 1998). Similar behavioral changes were observed in other passerine migrants (Beason et al., 1995, 1997; W. Wiltschko and Wiltschko, 1995; Beason and Semm, 1996). However, this effect was restricted to experienced birds that had already completed at least one migration trip; naive birds tested during their first migratory season remained unaffected and continued in their migratory direction (Munro et al., 1997a,b). The latter finding suggested that the effect of the pulse must involve an experience-dependent system and, together with the responses to changes in intensity recorded from the ophthalmic nerve (Beason, 1989; Semm and Beason, 1990), led to the conclusion that a possible magnetite-based receptor provides magnetic 'map' information by measuring intensity and is part of a system indicating position (for discussion, see W. Wiltschko and Wiltschko, 1998). The experiments by Beason and Semm (1986) also suggested that the pulse affected the 'map', not the compass. In displaced homing pigeons, the pulse effect was less pronounced and more variable, which is attributed to the multi-factorial nature of the pigeons' navigational 'map' (Beason et al., 1997).

The experimental procedures applied in these experiments were criticized by one of us (J.K.) for reasons given below. So we decided to collaborate and perform the study described here, where we modified the experimental technique in order to obtain a more predictable effect on the magnetite particles involved in a possible receptor.

\section{Theoretical background of the experiments}

It is important here to briefly review the physical principles behind our pulse remagnetization experiment. Magnetite is the only known ferromagnetic (or, more precisely, ferrimagnetic) substance of biogenetic origin in animals. Magnetite particles of single domain size have a stable magnetic moment aligned along their long particle axis. In magnetotactic bacteria, chains of single domains, now termed magnetosome chains, were described by Blakemore (1975) and later identified as magnetite by Kirschvink and Lowenstam (1979) and Blakemore (1982). Individual crystals are kept aligned both by magnetic interactions, which hold the magnetic moment of the individual magnetite crystals parallel to the chain axis, and by an organic supporting matrix (Kirschvink, 1982). In a linear chain, the moments simply add vectorially along the chain axis to maximize the magnetic moment and torque. However, the application of an external magnetic field can force the moment of each crystal to rotate slightly relative to the crystal axis. At the same time, this external magnetic field will also put a mechanical torque on the crystals, and the entire chain will attempt to rotate into alignment with it, which is how a compass works. If the external magnetic field exceeds a certain strength, that is, if it is strong enough to overcome the anisotropy energy, and if it is applied so that is antiparallel to the magnetic direction of crystals, the magnetization can be permanently reversed. For this to happen, however, the field must be applied rapidly enough so that the magnetosome chain does not have time to rotate mechanically with the field.

Kalmijn and Blakemore (1978) reported the first pulse remagnetization experiments on the magnetotactic bacteria. To guarantee that the magnetic pulse was aligned so that it was antiparallel to the magnetic direction of the bacteria, they first used a static magnetic field (approximately $1 \mathrm{mT}$; about 20 times stronger than the geomagnetic field) to align the bacteria and then applied a brief pulse (approximately $1 \mu \mathrm{s}$ ). Strong pulses antiparallel to the direction of this biasing field were able to completely reverse the swimming direction of all bacteria present, indicating that the magnetosomes had been remagnetized in the reversed direction, whereas pulses parallel to the bias field had no effect (Diaz-Ricci at al., 1991).

In experiments with birds (Wiltschko et al., 1994, 1998; Beason et al., 1995, 1997), the situation was different. The only 'biasing field' present before and during treatment was the much weaker geomagnetic field. The birds were magnetized 'south anterior', as defined by Beason et al. (1995), while facing east, that is, the pulse was applied perpendicular to that field. The very fact that the pulse remagnetization experiments did have an effect on the birds' behavior argues strongly that a magnetite-based receptor of some sort is involved, as no other known biophysical mechanism for transducing the geomagnetic field to the nervous system would show any effect after pulse treatment. However, the observed $90^{\circ}$ change in direction did not allow any conclusions about the nature and specific arrangement of magnetite with the receptor system.

Mann et al. (1988) isolated chains of single domain particles from the ethmoid region of sockeye salmon Oncorhynchus nerka and, more recently, Walker et al. (1997) and Diebel et al. (2000) described chains of single domains in the lamina propria layer within the olfactory lamellae of the rainbow trout Oncorhynchus mykiss. As magnetic measurements (e.g. Beason and Nichols, 1984; Beason and Brennon, 1986; Edwards et al., 1992) had indicated the presence of single 
domains also in the heads of birds (see also Kirschvink and Walker, 1986), it seemed reasonable to assume that similar chains of single domain magnetite are part of a possible magnetoreceptor. If such magnetosome chains were free to move to some extent, a pulse perpendicular to a weak biasing field is expected to remagnetize roughly half of the magnetosomes, and may actually produce some heterogeneously magnetized chains, causing some of them to kink or bend. The effect of the pulse on a possible receptor structure could thus not be clearly defined. This was pointed out by one of us (J.K.), who suggested that we use a procedure similar to that by Kalmijn and Blakemore (1978) on the magnetotactic bacteria. The 'bacteria-like' configuration is clearly the simplest hypothesis for the construction of a magnetite-based receptor (e.g. Kirschvink and Gould, 1981) and lends itself to similar tests via pulse-remagnetization: if the magnetite particles in a receptor were single domains arranged in magnetosome chains that were free to move, a strong biasing field could be used to rotate and reliably align them in the direction of that field. Application of a pulse antiparallel to that direction should remagnetize them all, thus producing a maximum effect, whereas a pulse parallel to that direction would have little or no effect. The present study was designed to test whether the above prediction was correct.

\section{Materials and methods}

The experiments took place in Sydney, NSW, Australia $\left(33^{\circ} 37^{\prime} \mathrm{S}, 151^{\circ} 10^{\prime} \mathrm{E}\right)$, during austral autumn from 7 to 25 March, 1998.

\section{Test birds}

As in the previous studies, the test birds were Australian silvereyes of the migratory Tasmanian population, Zosterops l. lateralis (Latham). These birds migrate in flocks predominantly during the twilight hours at dawn and dusk (Lane and Battam, 1971; Chan, 1995); they cross Bass Straight to spend their winter on the Australian continent.

The test birds were captured in Hobart, Tasmania, between 1 and 3 February, 1998. They were aged according to scull ossification (Pyle et al., 1987) and plumage characteristics (A. Leishman, personal communication). Twenty adult individuals were selected for the present study. The birds were transferred by airplane to Sydney and housed in a laboratory building on the Kuring-gai campus of the University of Technology, Sydney.

\section{Test conditions and performance}

All tests took place in a wooden hut at the Kuring-gai campus in the local geomagnetic field $\left(57500 \mathrm{nT},-64^{\circ}\right.$ inclination). Testing followed a standard sequence: it began with a series of six control tests to determine the directional preference of each individual in order to assure that the birds showed appropriate migratory behavior and to document the stability of the directional choices from day to day. A single test performed immediately after exposure to a biasing field was to check whether this field by itself would affect behavior. Two critical tests after treatment with the pulse in the presence of the biasing field completed the sequence, the first one starting immediately after treatment, the second one taking place the following day.

In view of these critical tests, the birds were subdivided into two groups, PAR and ANTI, according to the direction of the biasing field in relation to the direction of the pulse. One bird escaped during the initial phase; therefore, the group ANTI consisted of nine birds and group PAR of ten birds.

The biasing field was $1 \mathrm{mT}$, approximately 20 times the earth field's intensity, produced by Helmholtz coils, with magnetic north in geographic east for the ANTI-birds and in geographic west for the PAR-birds. It added to the local geomagnetic field so that the north directions of the combined fields deviated from east and west, respectively, by about $2^{\circ}$ towards north. The birds were exposed to the combined field for about $5 \mathrm{~s}$ while facing geographic east, which meant magnetic north for the ANTI-birds and magnetic south for the PAR-birds. The pulse, of intensity $0.5 \mathrm{~T}$ and duration approximately $4-5 \mathrm{~ms}$, was identical to the one used in earlier studies and was administered in the same way (W. Wiltschko et al., 1994, 1998), being 'south anterior' as defined by Beason et al. (1995, 1997). This pulse was applied while the birds were exposed to the $1 \mathrm{mT}$ biasing field oriented as before, which means that the pulse was parallel to the field for the PAR-birds and antiparallel for the ANTI-birds.

All the birds in each group were tested simultaneously every second day, except for the critical tests that took place on 2 consecutive days. Testing started approximately $30 \mathrm{~min}$ before the lights went off in the housing room and ended after approximately $75 \mathrm{~min}$. The light level of the diffuse light in the test cages was about $51 \mathrm{x}$.

\section{Data recording and analysis}

Orientation was recorded in funnel cages (see Emlen and Emlen, 1966) lined with typewriter correction paper (Bic, formerly Tipp-Ex, Germany). The birds were tested one in each cage; they left scratches in the coating of the inclined wall when they moved. For evaluation, the paper was removed, divided into 24 sectors, and the number of scratches in each sector counted. Recordings with fewer than 35 scratches were excluded because of insufficient migratory activity.

From the distribution of activity, we calculated the heading of each recording. To characterize the behavior during the control phase, we calculated the individual birds' mean vectors from up to six control headings per bird, and, on the basis of the mean headings, calculated grand mean vectors for the two groups by vector addition. To assess the effect of the treatment, mean vectors were calculated from the headings recorded (up to 10) each testing day, which were tested using the Rayleigh test for directional preference. The axial vector was obtained by doubling the angles and retransformation (see Batschelet, 1981).

The behavior of the two groups was compared using the Mardia-Watson-Wheeler test (Batschelet, 1981) on the 


\section{W. Wiltschko and others}

original distribution and the distribution of the doubled angles. With the same test, we also compared the behavior of the birds after exposure to the bias field and pulse treatment with that during the control phase before treatment.

\section{Results}

Fig. 1 presents the orientation behavior of the test birds recorded during the various phases of the test sequence; the vectors for both groups on consecutive testing days are given in Table 1.

The well oriented behavior of our test birds in their northerly migratory direction during the control phase is obvious. Most individual birds have long vectors (see Fig. 1A). The grand mean vectors based on the birds' mean headings, with $11^{\circ}, 0.98$ for the PAR-group and $2^{\circ}, 0.87$ for the ANTI-group, were both highly significant $(P<0.001$, Rayleigh test). The ANTI-group appears to show slightly more scatter; statistically, however, the two groups do not differ from each other before treatment $(P>0.05$; Mardia-Watson-Wheeler test). Exposure to the biasing field of $1 \mathrm{mT}$ alone did not have a noticeable effect on the orientation behavior (Fig. 1B); the directional preferences did not differ from those in the control phase $(P>0.05$ for both groups and all six control days).

After treatment with the pulse in the presence of the biasing field, both groups changed their behavior (PAR: $P<0.05$, all control days except day 2; ANTI: $P<0.05$, all control days except day 6). The birds were no longer oriented in their migratory direction, but showed a significant preference for an axis that roughly coincided with the east-west axis (Fig. 1C), which was more pronounced on the second day after treatment, with more headings in the westerly direction. Again, there was no difference between the PAR birds and the ANTI birds, both groups showing the same pattern $(P>0.05$; see Table 1$)$. This means that the direction of the pulse with respect to the biasing field had no effect on the response.

It is impossible to decide whether the observed response represents a preference for the magnetic east-west axis or a preference for an axis perpendicular to the migration axis. With the migratory direction being so close to north, the confidence intervals include both axes.

\section{Discussion}

Although the biasing field alone did not affect behavior, the magnetic pulse, as before, had a marked after-effect on the orientation of Australian silvereyes. Their bimodal response to the pulse administered in the presence of the biasing field is somewhat different from the unimodal tendencies towards the eastward end of this axis observed in two previous studies (W. Wiltschko et al., 1994, 1998). In view of the hypothesis tested, however, it is clear that the effect was not as we had expected under the assumption of mobile, polarity-sensitive magnetosome chains. If such chains were part of the magnetoreceptor involved in migratory behavior, one would have predicted a marked difference in the responses of the two
A
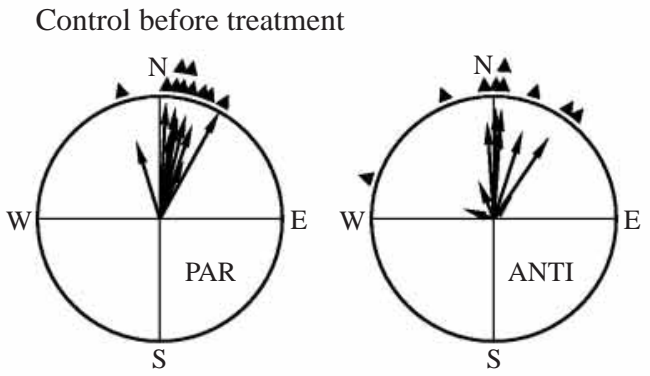

B After treatment with biasing field
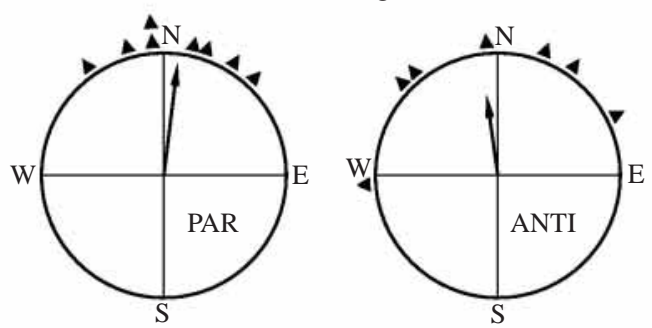

C

After treatment with pulse

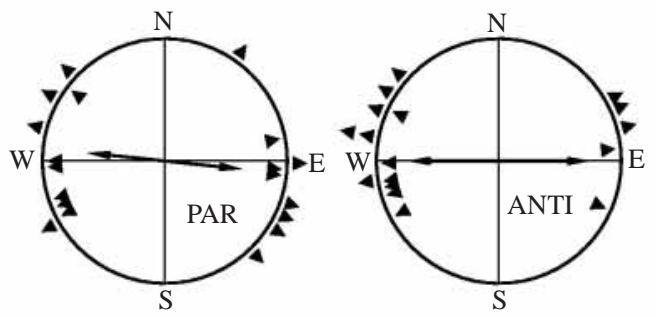

Fig. 1. Orientation behavior of silvereyes during the control phase (A) after exposure to the biasing field of $1 \mathrm{mT}$ (B) and after treatment with a brief, strong magnetic pulse in the presence of the same biasing field (C). Left: PAR birds where the pulse was parallel to the biasing field; right: ANTI birds where the pulse was antiparallel to the biasing field. In A, the arrows represent mean vectors of up to six recordings per individual bird, drawn proportionally to the radius of the circle; the symbols at the periphery mark the mean headings. In B and $\mathrm{C}$, symbols at the periphery represent headings of individual tests (in $\mathrm{C}$ : outside=day 1; inside=day 2), and the arrows give the axial vector of the respective data.

groups, with ANTI birds showing a much larger response than PAR birds. This is obviously not what we observed. Instead, the birds responded in the same way, regardless of the direction of the biasing field. Our findings thus argue against the initial hypothesis of freely moving single domain particles being part of a polarity-sensitive, magnetite-based receptor.

What type of magnetoreceptor is suggested by the response of the birds and, respectively, what type of receptor is compatible with our results? One possibility is that a magnetoreceptor contains single domains with limited freedom of motion under normal conditions, so that some of them had not been aligned in the biasing field and, as a consequence, were remagnetized in the PAR situation. Magnetoreceptors, which have evolved to extract an intensity signal from the geomagnetic field, do not necessarily need to have a full range 
Table 1. Orientation of silvereyes before and after treatment with a brief, strong magnetic pulse administered together with a biasing field

\begin{tabular}{|c|c|c|c|c|c|c|c|}
\hline & \multicolumn{3}{|c|}{ PAR birds } & \multicolumn{3}{|c|}{ ANTI birds } & $\begin{array}{c}\text { Significant } \\
\text { difference }\end{array}$ \\
\hline \multicolumn{8}{|l|}{ Control } \\
\hline Day 2 & 8 & $5^{\circ}$ & $0.62 *$ & 8 & $11^{\circ}$ & $0.69 *$ & NS \\
\hline Day 3 & 8 & $23^{\circ}$ & $0.85^{* *}$ & 8 & $352^{\circ}$ & $0.67 *$ & NS \\
\hline Day 4 & 10 & $11^{\circ}$ & $0.84 * *$ & 8 & $357^{\circ}$ & $0.66^{*}$ & NS \\
\hline Biasing field & 9 & $7^{\circ}$ & $0.92 * * *$ & 7 & $352^{\circ}$ & $0.66^{*}$ & NS \\
\hline \multicolumn{8}{|c|}{ After treatment with the pulse in the presence of biasing field: } \\
\hline Day of treatment & 10 & $95^{\circ}$ & 0.16 & 9 & $317^{\circ}$ & 0.45 & NS \\
\hline Axial: & & $111-291^{\circ}$ & $0.59 *$ & & $94-274^{\circ}$ & $0.69 *$ & NS \\
\hline
\end{tabular}

of motion (for a discussion, see Kirschvink and Gould, 1981; Kirschvink and Walker, 1985). A magnetic pulse, applied to magnetosomes that are not free to move, is likely to produce chains that are heterogeneously magnetized. It is impossible to say what the output of a receptor altered this way might mean to the birds; it could result in behaviors similar to those reported from the previous studies (W. Wiltschko et al., 1994, 1998) or observed in the present one.

Another possibility, however, is that the idea of an avian intensity receptor based on single domains arranged in chains might not be correct. One aspect of the previous pulse experiments (Wiltschko et al., 1994, 1998) that has always been hard to explain is the short duration of the effect. A clear effect was observed only on the day of treatment itself and the day after treatment; after a week of apparent disorientation, the migrants returned to their normal migratory direction (W. Wiltschko et al.,1994, 1998). Because remagnetization of single domains should be as stable as the original one, it is difficult to see how the previous state could be restored, and one must turn to auxiliary assumptions. One possibility is that some as-yet-unknown mechanisms allow the particles to gradually realign themselves in their original position during a week's interval of time, thus 'healing' and letting the birds recover their normal behavior. Single domain particles magnetized in opposite directions to the remainder of the chain would represent a high-energy state; hence it seems possible that the odd magnetite crystals rotate back into alignment within the receptor structure. This might lead to entire chains of particles magnetized in the opposite way from their original magnetization, but if it is only the magnitude of the magnetic moment of the particles, not their polarity, that determines the output of the receptor (see Kirschvink and Walker, 1985), this would not affect its functionality. By contrast, we cannot exclude the possibility that the fading of the effect may reflect a purely behavioral response. Although recalibration of a component of a position-locating system appears hardly possible as long as the birds are restricted to the small space of a cage (in contrast to pigeons that are released and home over considerable distances; see Beason et al., 1997), they might realize a continuing discrepancy between the input of a magnetite-based receptor and other cues involved in a multifactorial 'map'. This might cause them to simply ignore the information from that receptor, falling back on their innate migratory direction.

Histological studies looking for magnetite particles in birds have not yet produced clear evidence for single domains. Magnetosome chains, similar to those described in bacteria, have been found in fish, another vertebrate group (Mann et al., 1988; Diebel et al., 2000). In birds, single domains were indicated mostly by magnetic property measurements (Walcott et al., 1979; Beason and Nichols, 1984; Beason and Brennon, 1986; Edward et al., 1997); the isolated crystals of magnetite that were imaged (Kirschvink and Walker, 1986) had been extracted from the head of pigeons without any indication of their original position. Iron deposits in the tissue, in part associated with fibers of the ophthalmic nerve, have been identified using Prussian Blue (Beason and Nichols, 1984; Williams and Wild, 2001), but it has not been demonstrated whether they are in fact magnetite. In short, single domain crystals as such have not yet been demonstrated in their natural position embedded in the tissue as part of a receptor in birds, yet the existence of the relatively small numbers of single domains needed to explain the magnetoreceptive behavior cannot be excluded either. Hanzlik et al. (2000) and 
Winklhofer et al. (2001) recently identified magnetite particles of the much smaller size of superparamagnetic grains (SPM) in the skin of the upper beak of pigeons, that is, in the region of the receptive fields of the ophthalmic nerve. They were found within the subcutis, arranged in well defined clusters surrounded by a sheath of neurofilaments.

Since superparamagnetic crystals do not have stable magnetic moments, their magnetization remains unaffected by the pulse; similarly, the pulse would cause hardly any physical torque or translations, so that significant displacements of the crystals or mechanical damages of the receptor structure appear to be rather unlikely. In view of this, it is difficult to see how the pulse could affect a receptor based on superparamagnetic particles to cause the observed effect. Nevertheless, a pulse of about 10,000 times the earth field intensity represents an extremely strong signal to any receptor detecting magnetic intensity. The nature of the structures transmitting the signals is still unknown; a temporary impairment of a receptor built to record minute intensity differences cannot be totally excluded.

Our behavioral study is thus able to exclude the hypothesis that freely mobile chains of single domains are a component of the possible receptor, but it does not allow us to decide between the other possibilities. Little is known about the specific size of the magnetite particles in birds and in what structure they are embedded. So far, more detailed studies are only available for salmonid fish (Walker et al., 1997; Diebel et al., 2000). Recent work by Williams and Wild (2001) clearly indicates that the iron-containing structures in the beak of pigeons, although also innervated by a branch of the nervus trigeminus, are different in location and structure. Here, we must hope for new histological studies that identify magnetite particles in situ in the tissue of birds and at the same time show details of the receptor structure and connections to the nervous system.

Our work was supported by the Deutsche Forschungsgemeinschaft (W.W.) and a UTS internal research grant (U.M.). The experiments were performed according to the laws and regulations of Australia. We acknowledge the valuable help of A. Atkins, A. Fletcher, R. Rose and the Department of Zoology, University of Tasmania, with catching the test birds, and of L. Schultz with keeping the birds and performing the experiments. Thanks are also due to M. M. Walker, University of Auckland, New Zealand, and M. Winklhofer, Universität München, Germany, for useful discussions and comments.

\section{References}

Batschelet, E. (1981). Circular Statistics in Biology. New York: Academic Press.

Beason, R. C. (1989). Magnetic sensitivity and orientation in the bobolink. In Orientation and Navigation - Birds, Humans and other Animals. Proc. Int. Conf. Royal Inst Navig, Cardiff, Wales, paper 7.

Beason, R. C. and Brennan, W. J. (1986). Natural and indiced magnetization in the bobolink (Dolichonyx oryzivorus). J. Exp. Biol. 125, 49-56.

Beason, R. C. and Nichols, J. E. (1984). Magnetic orientation and magnetic sensitive material in a transequatorial migratory bird. Nature 309, 151-153.
Beason, R. C. and Semm, P. (1996). Does the avian ophthalmic nerve carry magnetic navigational information? J. Exp. Biol. 199, 1241-1244.

Beason, R. C., Dussourd, N. and Deutschlander, M. E. (1995). Behavioural evidence for the use of magnetic material in magnetoreception by a migratory bird. J. Exp. Biol. 198, 141-145.

Beason, R. C., Wiltschko, R. and Wiltschko, W. (1997). Pigeon homing: effects of magnetic pulses on initial orientation. Auk 114, 405-415.

Blakemore, R. P. (1975). Magnetotactic bacteria. Science 19, 377-379.

Blakemore, R. P. (1982). Magnetotactic bacteria. Ann. Rev. Microbiol. 36, 217-238.

Chan, K. (1995). Diurnal and nocturnal pattern of activity in resident and migrant Silvereyes Zosterops lateralis. Ети 95, 41-46.

Diaz-Ricci, J. C., Woodford, B. J., Kirschvink, J. L. and Hoffman, M. R. (1991). Alteration of the magnetic properties of Aquaspirillum magnetotacticum by a pulse magnetization technique. Appl. Environ. Microbiol. 57, 3248-3254.

Diebel, C. E, Proksch, R., Green, C. R., Neilson, P. and Walker, M. M. (2000). Magnetite defines a vertebrate magnetoreceptor. Nature 406, 299-302.

Edwards, H. H., Schnell, G. D., DuBois, R. L. and Hutchison, V. H. (1992). Natural and induced remanent magnetism in birds. Auk 109, 43-56.

Emlen, S. T. and Emlen, J. T. (1966). A technique for recording migratory orientation in captive birds. Auk 83, 361-367.

Gould, J. L., Kirschvink, J. L. and Deffeyes, K. S. (1978). Bees have magnetic remanence. Science 201, 1026-1028.

Gould, J. L., Kirschvink, J. L., Deffeyes, K. S. and Brines, M. L. (1980). Orientation of demagnetized bees. J. Exp. Biol. 86, 1-8.

Hanzlik, M., Heunemann, C., Holtkamp-Rötzler, E., Winklhofer, M., Petersen N. and Fleissner, G. (2000). Superparamagnetic magnetite in the upper beak tissue of homing pigeons. Biometals 13, 325-331.

Kalmijn, A. J. and Blakemore, R. P. (1978). The magnetic behavior of mud bacteria. In Animal Migration, Navigation and Homing (ed. K. SchmidtKoenig and W. T. Keeton), pp. 354-355. Berlin, Heidelberg, New York: Springer Verlag.

Kirschvink, J. L. (1982). Paleomagnetic evidence for fossil biogenic magnetite in western Crete. Earth Planet. Sci. Lett. 59, 388-392.

Kirschvink, J. L. (1989). Magnetite biomineralization and geomagnetic sensitivity in higher animals: an update and recommendations for future studies. Bioelectromagnetics 10, 239-259.

Kirschvink, J. L. and Gould, J. L. (1981). Biogenetic magnetite as a basis for magnetic field detection on animals. BioSystems 13, 181-201.

Kirschvink, J. L. and Kobayashi-Kirschvink, A. (1991). Is geomagnetic sensitivity real? Replication of the Walker-Bittermann magnetic conditioning experiments in honey bees. Amer. Zool. 31, 169-185.

Kirschvink, J. L. and Lowenstam, H. A. (1979). Mineralization and magnetization of chiton teeth: Paleomagnetic, sedimentologic, and biologic implications of organic magnetite. Earth Planet. Sci. Lett. 44, 193-204.

Kirschvink, J. L. and Walker, M. M. (1985). Particle-size considerations for magnetite-based magnetoreceptors. In Magnetite Biomineralization and Magnetoreception in Organisms (ed. J. L. Kirschvink, D. S. Jones and B. J. MacFadden), pp. 243-256. New York: Plenum Press.

Kirschvink, J. L. and Walker, M. M. (1986). Biogenic magnetite in higher organisms and the current status of the hypothesis of ferrimagnetic magnetoreception. In Biophysical Effects of Steady Magnetic Fields (ed. G. Maret, N. Boccara and J. Kiepenheuer), pp. 180-188. New York: Springer Verlag.

Kirschvink, J. L., Jones, D. S. and MacFadden, B. J. (1985). Magnetite Biomineralization and Magnetoreception in Organisms. New York: Plenum Press.

Kirschvink, J. L., Padmanabha, S., Boyce, C. K. and Oglesby, J. (1997). Measurement of the threshold sensitivity of honeybees to weak, extremely low frequency magnetic fields. J. Exp. Biol. 200, 1363-1368.

Lane, S. G. and Battam, H. (1971). Silvereye movement in Eastern Australia. Aust. Bird Bander 9, 80-82.

Lowenstam, H. A. (1962). Magnetite in denticle capping in recent chitons (Polyplacophora). Geol. Soc. Am. Bull. 73, 435-438.

Mann, S., Sparks, N. H. C., Walker, M. M. and Kirschvink, J. L. (1988). Ultrastucture, morphology and organization of biogenic magnetite from Sockeye Salmon, Oncorhynchus nerka: implications for magnetoreception. J. Exp. Biol. 140, 35-49.

Munro, U., Munro, J. A., Phillips, J. B., Wiltschko, R. and Wiltschko, W. (1997a). Evidence for a magnetite-based navigational 'map' in birds. Naturwissenschaften 84, 26-28.

Munro, U., Munro, J. A., Phillips, J. B. and Wiltschko, W. (1997b). 
Effect of wavelength of light and pulse magnetisation on different magnetoreception systems in a migratory bird. Austr. J. Zool. 45, 189-198.

Pyle, P., Howell, S. N. G., Yunick, R. P. and DeSante, D. F. (1987). Identification Guide to North American Passerines. Slate Creek Press: Bolinas.

Semm, P. and Beason, R. C. (1990): Responses to small magnetic variations by the trigeminal system in the bobolink. Brain. Res. Bull. 25, 735-740.

Shcherbakov, V. P. and Winklhofer, M. (1999). The osmotic magnetometer: a new model for magnetite-based magnetorecepters in animals. Eur. Biophys. J. 28, 380-392.

Walcott, C., Gould, J. L. and Kirschvink, J. L. (1979). Pigeons have magnets. Science 205, 1027-1029.

Walcott, C., Gould, J. L. and Lednor, J. L. (1988). Homing of magnetized and demagnetized pigeons. J. Exp. Biol. 134, 27-41.

Walker, M. M., Kirschvink, J. L. and Dizon, A. E. (1985). Magnetoreception and magnetite biomineralization in fish. In Magnetite Biomineralization and Magnetoreception in Organisms: A New Biomagnetism, vol. 5 (ed. J. L. Kirschvink, D. S. Jones and B. McFadden), pp. 417-437. New York: Plenum Press.

Walker, M. M., Diebel, C. E., Haugh, C. V., Pankhurst, P. M., Montgomery, J. C. and Green, C. R. (1997). Structure and function of the vertebrate magnetic sense. Nature 390, 371-376.
Williams, M. N. and Wild, J. M. (2001). Trigeminally innervated ironcontaining structures in the beak of homing pigeons, and other birds. Brain Res. 889, 243-246.

Wiltschko, R. and Wiltschko, W. (1995). Magnetic Orientation in Animals. Berlin: Springer Verlag.

Wiltschko, W. and Wiltschko, R. (1995). Migratory orientation of European robins is affected by the wavelength of light as well as by a magnetic pulse. J. Comp. Physiol. A 177, 363-369.

Wiltschko, W. and Wiltschko, R. (1998). The navigation system of brids and its development. In Animal Cognition in Nature (ed. R. P. Balda, I. M. Pepperberg and A. C. Kamil), pp. 155-199. San Diego: Academic Press.

Wiltschko, W., Munro, U., Beason, R. C., Ford, H. and Wiltschko, R. (1994). A magnetic pulse leads to a temporary deflection in the orientation of migratory birds. Experientia 50, 697-700.

Wiltschko, W., Munro, U., Ford, H. and Wiltschko, R. (1998). Effect of a magnetic pulse on the orientation of Silvereyes, Zosterops l. lateralis, during spring migration. J. Exp. Biol. 201, 3257-3261.

Winklhofer, M., Holtkamp-Rötzler, E., Hanzlik, M., Fleisser, G. and Petersen, N. (2001). Clusters of superparamagnetic magnetite particles in the upper beak skin of homing pigeons: evidence of a magnetoreceptor? Eur. J. Mineral. 13, 659-669.

Yorke, E. D. (1979). A possible magnetic transducer in birds. J. Theor. Biol. 77, 101-105. 